\title{
Análise das causas de atendimento e prevalência das doenças oculares no serviço de urgência
}

\author{
The causes and prevalence of medical attendance \\ for ocular diseases in an emergency
}

Maria Nice Araujo Moraes Rocha', Marcos Ávila², David Leonardo Cruvinel Isaac ${ }^{3}$, Laís Leão de Oliveira ${ }^{4}$, Luísa Salles de Moura Mendonça ${ }^{4}$

\begin{abstract}
RESUMO
Objetivo: Identificar as doenças oculares mais comumente presentes no serviço de urgência de um Centro de Referência em Oftalmologia localizado na região Centro-Oeste do Brasil. Métodos: Estudo de delineamento transversal, observacional, retrospectivo com análise de prontuários de atendimentos realizados no período de abril de 2009 a março de 2010, no Centro de Referência em Oftalmologia - CEROF da Universidade Federal de Goiás. As variáveis estudadas foram idade, sexo, procedência, doenças oculares diagnosticadas e doenças sistêmicas associadas. Resultados: Um número total de 2105 prontuários foram analisados neste estudo. A faixa etária mais encontrada foi entre 15 e 29 anos (30,2\%), seguida pela entre 30 e 39 anos (20,9\%) e entre 40 e 49 anos $(14,9 \%)$, a idade média foi 34,3 anos. Houve predominância de pacientes do sexo masculino $(64,8 \%)$ em relação ao feminino $(35,2 \%)$. O local de procedência predominante foi o município de Goiânia (80,7\%), com 19,2\% de pacientes do interior do estado e de outros estados. As maiores causas de atendimento foram: corpo estranho extraocular $(25,5 \%)$, conjuntivites infecciosas (23,6\%) e ceratites e úlceras de córnea(11,3\%), tumores de pálpebras e órbita(7,2\%), olho seco(4,7\%), e blefarite(3,8\%). O trauma ocular registrou prevalência de 6,3\%.Conclusão: As doenças oculares atendidas mais frequentemente na urgência do CEROF são semelhantes a outros serviços da mesma natureza no Brasil. A maior parte dos casos é considerada de resolução simples, que podem ser tratados em serviços de atendimento primário, reduzindo a referência a serviços de atendimento terciário delegando a estes casos de maior complexidade.
\end{abstract}

Descritores: Oftalmopatias/epidemiologia; Conjuntivite; Traumatismos oculares; Emergências; Prevalência

\begin{abstract}
Objective: To identify the prevalence of ophthalmic diseases seen in the emergency department of a specialized center in Brazilian CenterWestern area. Methods: This is a retrospective transverse observation study based on patients chart who were seen at Ophthalmic Reference Center (CEROF), Federal University of Goiás, Goiânia (GO) Brazil, between april, 2009 to march, 2010. Age, gender, origin, ocular and associated systemic diseases were identified. Results: 2105 charts were analyzed. The age of patients ranged between 15-29 years-old $(30.2 \%)$, 30-39 years-old $(20.9 \%)$ and $40-49$ years-old $(14.9 \%)$, with a 34.3 years-old on average. Male gender were more frequent than females (64.8\% vs 35.2\%). The majority of patients live in Goiania (80.7\%) and the remaining (19.3) came from other cities in the county area or from other states in Brazil. Ocular dust was the leading cause for medical attendance, followed by infectious conjunctivitis $(23.6 \%)$, keratitis and cornea ulcer (11.3\%), tumors of the eyelids or the orbit $(7.2 \%)$, dry eyes $(4.7 \%)$, and blepharitis $(3.8 \%)$. Ocular trauma occurred in a $6.3 \%$ of patients. Conclusion: Ocular diseases in patients presenting at the emergency department of an ophthalmic reference center in the Center-Western area have a similar prevalence as in other centers in Brazil. Most of them can be treated in common health facilities and don't need to be referred to specialized centers. These centers should deal with more complex problems, saving costs and time.
\end{abstract}

Keywords: Eyes diseases; /epidemiology; Conjunctivitis; Eye injuries; Emergencies; Prevalence

\footnotetext{
${ }^{1}$ Pós-graduanda (Mestrado) do Programa de Pós-graduação (Mestrado) em Ciências da Saúde da Universidade Federal de Goiás (UFG) Goiânia(GO), Brasil;

${ }^{2}$ Universidade Federal de Goiás (UFG) - Goiânia(GO), Brasil;

${ }^{3}$ Universidade Federal de Goiás (UFG) - Goiânia (GO), Brasil;

${ }^{4}$ Acadêmicas do curso de Medicina da Universidade Federal de Goiás (UFG) - Goiânia (GO), Brasil.
}

Trabalho realizado no Centro de Referência em Oftalmologia, Universidade Federal de Goiás (UFG) - Goiânia (GO), Brasil.

Os autores declaram não haver conflitos de interesse

Recebido para publicação em: 13/10/2011 - Aceito para publicação em: 15/7/2012

Rev Bras Oftalmol. 2012; 71 (6): 380-4 


\section{INTRODUÇãO}

$\mathbf{N}$ os hospitais universitários e em serviços de atendimento terciário em oftalmologia, os serviços de urgência oftalmológica representam importante parte do atendimento a pacientes com afecções oculares. Estes serviços são, em muitos casos, a porta de entrada e contato inicial de pacientes com um serviço oftalmológico, sendo atendidas, além de urgências propriamente ditas, diversas outras doenças oculares de pacientes que procuram estes serviços como atendimento inicial.

O Centro de Referência em Oftalmologia - CEROF é parte integrante da Faculdade de Medicina da Universidade Federal de Goiás (UFG) e atende pacientes em parceria com a Secretaria Municipal de Saúde através do Sistema Único de Saúde (SUS).

O conhecimento da prevalência no atendimento de urgência oftalmológica é de extrema importância, pois possibilita fornecer informações para o planejamento de estratégias preventivas, estabelecimento de políticas de saúde e análise e direcionamento do serviço onde se realizou a pesquisa.

O oftalmologista deve estar preparado para um diagnóstico correto e tratamento apropriado de urgências oculares por serem questões importantes para um prognóstico visual que pode resultar em cegueira ${ }^{(1)}$. Estudos mostram que 5 a $82 \%$ das consultas no departamento de emergência são por problemas eletivos. Esta larga variação se deve ao desconhecimento da população para definir um critério de urgência.

As causas mais comuns de atendimento na urgência ocu$\operatorname{lar}^{(1-4)}$ são traumas oculares, infecções oculares, tumores, descolamento de retina e uveíte.

O trauma ocular tem importante impacto na saúde ocular do paciente e socioeconômico no sistema de saúde. Dados da Organização Mundial de Saúde (OMS) revelam que a cada ano cerca de 55 milhões de traumatismos oculares são responsáveis por perda de dias de trabalho ${ }^{(2)}$.

Segundo Goiato $^{(4)}$, os traumas oculares são importantes causas de perda ocular, sendo $42 \%$ de origem ocupacional (em torno de 1 milhão de acidentes de trabalho oculares/ano). O trabalho relata que a média de traumas ocupacionais atingindo o olho na Inglaterra é de $45-52 \%$ e no Brasil, $10 \%$. Estudos em pronto-socorro de referência em hospital universitário do sul do Brasil referem que os trabalhadores mais acometidos foram os operários das indústrias, serralheiros e agricultores e o uso de óculos de proteção foi baixo nos traumas oculares por acidentes de trabalho ${ }^{(2)}$.

Na região sul do Brasil estudos relatam que além dos traumas, as inflamações palpebrais, os transtornos de conjuntiva, da córnea e esclera foram os diagnósticos predominantes, dentre os quais, o corpo estranho de córnea, as ceratites superficiais, a conjuntivite, o pterígio, a blefarite são causas frequentes de procura ao oftalmologista na urgência ${ }^{(5-6)}$.

Este estudo tem por objetivo identificar as doenças oculares mais prevalentes, atendidas no setor de urgência em um Hospital de referência em oftalmologia localizado na região Centro-oeste do Brasil.

\section{Métodos}

Foi realizado um estudo de corte transversal, retrospectivo envolvendo 2.105 prontuários de pacientes atendidos no ambulatório de urgência do Centro de Referência em Oftalmologia do Hospital das Clínicas da Universidade Federal de Goiás (CEROF/UFG), através do Sistema Único de Saúde (SUS), representando uma parcela de Goiás e Centro-Oeste brasileiro, no período de abril de 2009 a março de 2010. O estudo foi aprovado pelo Comitê de Ética do Hospital das Clínicas da Universidade Federal de Goiás. Os atendimentos foram realizados por oftalmologistas e residentes do serviço e registrados em prontuários padronizados para a instituição.

A amostra de 2105 pacientes foi calculada com base no total de 8689 pacientes atendidos na urgência do hospital no período estudado (Intervalo de Confiança de $95 \%$ e erro de estimativa de 0,05 ). Foram incluídos prontuários de pacientes atendidos na urgência, sem discriminação pelo sexo ou idade e excluídos prontuários com registros incompletos ou ilegíveis para análise. A amostra foi calculada pelo modelo matemático de proporção de acordo com o atendimento mensal na urgência, sendo os prontuários selecionados de forma seqüencial no arquivo do Hospital, até completar o número programado.

Foram analisados os dados de identificação do paciente (idade, sexo, procedência) e do exame oftalmológico (anamnese, antecedentes pessoais e familiares, biomicroscopia de câmara anterior, tonometria de aplanação, fundoscopia e diagnóstico conclusivo). Os dados foram registrados em planilhas e as doenças categorizadas em 10 grupos de acordo com o diagnóstico final. A idade dos pacientes foi dividida em sete faixas etárias: 0 a 14,15 a 29,30 a 39,40 a 49,50 a 59,60 a 69 e $\geq 70$ anos. A procedência foi classificada em 3 categorias: pacientes provenientes de Goiânia, do interior do estado de Goiás e de outros estados do Brasil.

A categorização das doenças foi baseada (Tabela1) na região anatômica atingida do olho, exceto para as categorias cefaléia e trauma por se tratarem de dados referentes a sintomas e meio provocador de lesão ocular, respectivamente.

Tabela 1

Categorias de doenças oculares no ambulatório de urgência

\begin{tabular}{ll}
\hline \multicolumn{1}{c}{ Categorias } & \multicolumn{1}{c}{ Doenças oculares } \\
\hline Córnea, conjuntiva e esclera & $\begin{array}{c}\text { Corpo estranho extraocular, Conjuntivite infecciosa, Ceratite e Úlcera de córnea, Pterígio/ } \\
\text { Pingueculite, Hemorragia subconjuntival, Conjuntivite alérgica, Episclerite/esclerite, Leucoma, } \\
\text { Tumor de conjuntiva } \\
\text { Tumores palpebrais e orbitários, Olho seco, Blefarite, Triquíase, Calázio, Alterações de vias } \\
\text { lacrimais / Dacrioadenite, Ptose palpebral, Lagoftalmo }\end{array}$ \\
$\begin{array}{l}\text { Trauma ocular } \\
\text { Queimaduras }\end{array}$ & Químicas, Actínicas ou térmicas \\
$\begin{array}{l}\text { Uveíte } \\
\text { Globo ocular e órbita } \\
\text { Catarata } \\
\text { Glaucoma } \\
\text { Cefaléia }\end{array}$ & Atrofia bulbar, Celulite orbitária, Hematoma periorbitário \\
\hline
\end{tabular}


Os programas utilizados foram o Excel 7(Microsoft) para processamento dos dados e os resultados analisados estatìsticamente pelos programas SPSS for Windows, versão 13.0 expressos em frequência absoluta e relativa (porcentagem).

\section{RESULTADOS}

A amostra de prontuários do pronto socorro do CEROF correspondeu a 2105 casos $(24,23 \%)$ de um total de 8689 prontuários de consultas atendidas no período citado (Figura 1).

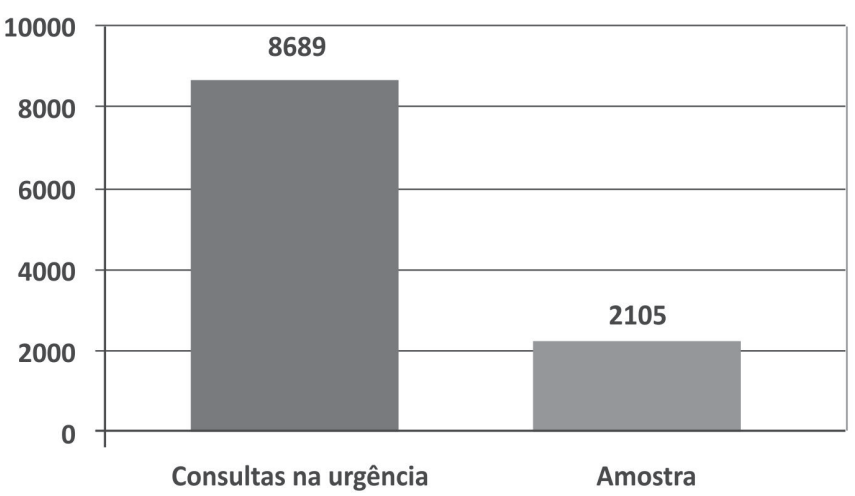

Fonte: Prontuários do CEROF/UFG Modelo matemático de proporção

Figura1: Proporção entre o total de consultas atendidas na urgência e amostra

Analisando a demanda atendida na urgência por trimestres encontramos predominância no terceiro trimestre (645$30,92 \%$ ), seguido pelo segundo (542- $26,63 \%$ ) e primeiro (514$24,30 \% 0$ trimestres, sendo o quarto trimestre (404-19,10\%) o de menor procura (Figura 2).

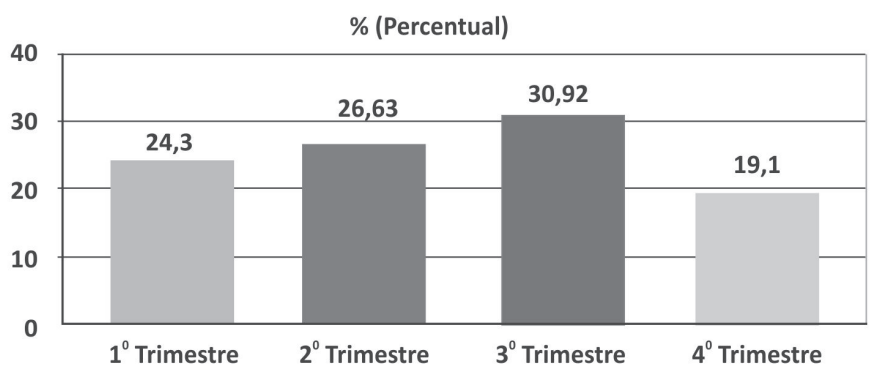

Fonte: Prontuários do CEROF/UFG modelo matemático de proporção

Figura 2: Distribuição das consultas de urgência por trimestre

Do total da amostra 51,06\% tinham entre 15 e 39 anos e a menor freqüência foi registrada em pacientes entre 60 a 69anos $(5,46 \%)$ e $\geq 70$ anos $(4,37 \%)$, seguida pela faixa entre 40 a 49 anos $(14,92 \%), 0$ a $14 \operatorname{anos}(13,3 \%)$ e 50 a $59 \operatorname{anos}(10,88 \%)$ (Figura3).

A idade média dos pacientes atendidos foi 34,31 anos (mínima de 0 a 1 mês e máxima de 100 anos), com 191 idosos com idade média de 70,38 anos (mínima de 61 e máxima de 89 anos).

As crianças até 14 anos completos representaram 13,3\% (280 casos) da amostra atendida na urgência.

O sexo masculino teve maior atendimento (1365 casos$64,84 \%$ ) em relação ao feminino ( 740 casos - $35,15 \%$ ), conforme resultados mostrados na Figura 4.

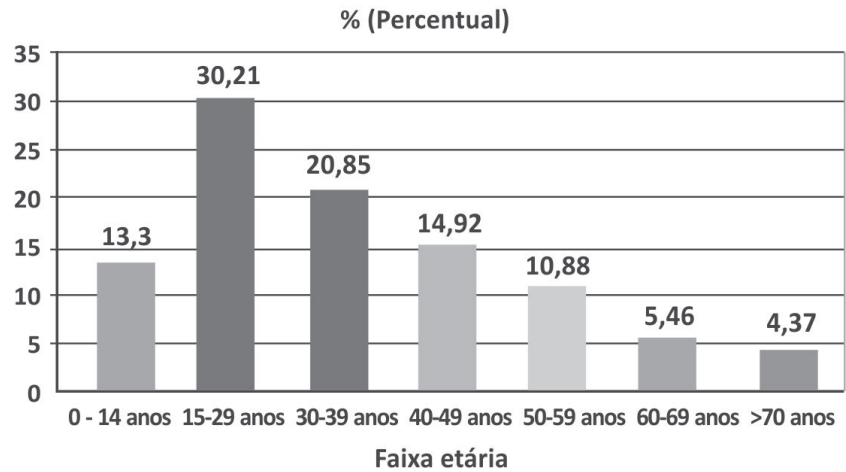

Fonte: Prontuários do CEROF/UFG modelo matemático de proporção

Figura 3: Distribuição das consultas na urgência por faixa etária

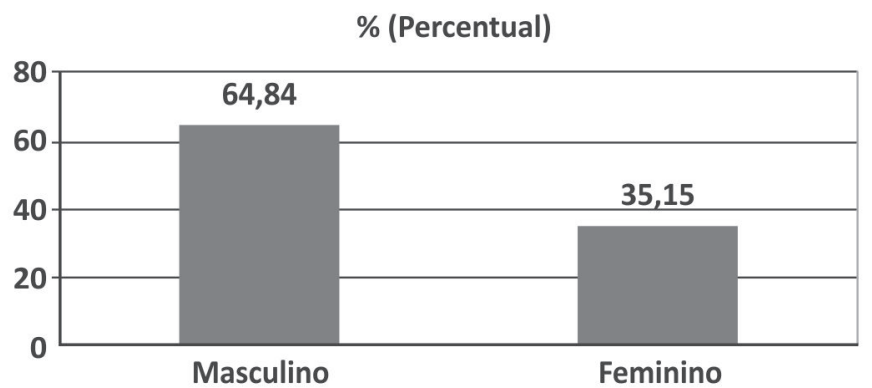

Fonte: Prontuários do CEROF/UFG modelo matemático de proporção

Figura 4: Distribuição das consultas na urgência por gênero

A maioria dos pacientes informou domicílio em Goiânia $(80,7 \%)$, com menor procedência do interior do estado de Goiás $(18,9 \%)$ e outros estados $(0,2 \%)$.

$\mathrm{Na}$ amostra de 2105 consultas na urgência, as categorias de doenças da córnea, conjuntiva e esclera tiveram uma parcela expressiva em atendimento de urgência com 1508 casos (71,7\%), seguidas pelo grupo das doenças palpebrais e sistema lacrimal com 378 casos $(18,5 \%)$ e o trauma ocular com 133 casos $(6,3 \%)$.

No grupo córnea, conjuntiva e esclera as prevalências maiores foram corpo estranho extraocular em 537 (25,5\%) casos, seguidos de conjuntivite infecciosa aguda, com 497 casos $(23,6 \%)$ e ceratites e úlceras de córnea com 237 (11,3\%). Estes três tipos de doenças totalizaram $1.271(60,4 \%)$, ressaltando em alguns casos, no mesmo paciente e até no mesmo olho houve casos com mais de uma doença. Dentre as doenças de pálpebras e sistema lacrimal, foram mais encontrados os tumores palpebrais e de órbita em 152 casos $(7,2 \%)$, olho seco com 99 casos $(4,7 \%)$, blefarite com 79 casos $(3,8 \%)$, triquíase com 20 casos $(1,0 \%)$ e calázio com13 casos $(1,1 \%)$. Os casos de obstrução de vias lacrimais e dacrioadenite apresentaram prevalência de $11 \mathrm{casos}$ $(0,5 \%)$.O trauma ocular registrou, na amostra, 133 casos $(6,3 \%)$ sendo mais comum o não penetrante provocado por contusão.As queimaduras oculares foram separadas em dois grupos, relacionadas ao agente causal: químicas, com 57 casos $(2,7 \%)$ e as físicas (actínicas, térmicas) com 30 casos $(1,4 \%)$. Os corpos estranhos foram mais localizados na córnea e em menor frequência na conjuntiva tarsal ou bulbar. Alguns apresentaram alterações corneanas associadas. 
As doenças menos prevalentes foram uveítes (não separados em tipos) que totalizaram 46 casos $(2,2 \%)$, doenças do globo ocular e órbita com 32 casos $(1,5 \%)$, seguidas pelas doenças de retina e vítreo com 29 casos $(1,4 \%)$ sendo 23 casos $(1,1 \%)$ de descolamento de retina e 6 casos $(0,3 \%)$ de hemorragia ví- trea. O glaucoma apresentou prevalência de 19 casos $(0,9 \%)$ e não foi classificado por tipo. $(0,6 \%)$.

A cefaléia foi motivo de consulta na urgência com 13 casos

Os dados estão sintetizados na Tabela 2.

Tabela 2

Distribuição dos diagnósticos nas consultas de urgência

\begin{tabular}{|c|c|c|c|}
\hline Diagnóstico & $\mathrm{n}^{\mathrm{o}}$ de casos & Percentual (\%) & IC (\%) \\
\hline Córnea, conjuntiva, esclera & 1508 & 71,63 & $(69,68-73,53)$ \\
\hline Corpo estranho extraocular & 537 & 25,51 & $(23,68-27,41)$ \\
\hline Conjuntivite infecciosa & 497 & 23,61 & $(21,83-25,46)$ \\
\hline Ceratite / úlcera de córnea & 237 & 11,26 & $(9,96-12,66)$ \\
\hline Pterígio / Pingueculite & 111 & 5,27 & $(4,38-6,29)$ \\
\hline Hemorragia subconjuntival & 84 & 3,99 & $(3,21-4,82)$ \\
\hline Conjuntivite alérgica & 17 & 0,80 & $(0,49-1,26)$ \\
\hline Episclerite /esclerite & 13 & 0,62 & $(0,34-1,03)$ \\
\hline Leucoma & 8 & 0,38 & $(0,18-0,72)$ \\
\hline Tumor de conjuntiva & 4 & 0,19 & $(0,07-0,46)$ \\
\hline Pálpebras e Sistema lacrimal & 378 & 17,96 & $(16,36-19,64)$ \\
\hline Tumores palpebrais e órbita & 152 & 7,22 & $(6,17-8,39)$ \\
\hline Olho seco & 99 & 4,70 & $(3,86-5,67)$ \\
\hline Blefarite & 79 & 3,75 & $(3,00-4,63)$ \\
\hline Triquíase & 20 & 0,95 & $(0,60-1,44)$ \\
\hline Calázio & 13 & 0,62 & $(0,34-1,03)$ \\
\hline Alteraçõess de vias lacrimais/Dacrioadenite & 11 & 0,52 & $(0,28-0,91)$ \\
\hline Ptose palpebral & 2 & 0,10 & $(0,02-0,31)$ \\
\hline Lagoftalmo & 2 & 0,10 & $(0,02-0,31)$ \\
\hline Trauma ocular & 133 & 6,32 & $(5,39-7,42)$ \\
\hline Queimaduras & 87 & 4,13 & $(3,34-5,05)$ \\
\hline Químicas & 57 & 2,71 & $(2,08-3,47)$ \\
\hline Actínicas & 30 & 1,43 & $(0,98-2,00)$ \\
\hline Uveíte & 46 & 2,19 & $(1,62-2,88)$ \\
\hline Globo ocular e órbita & 32 & 1,50 & $(1,06-2,14)$ \\
\hline Atrofia bulbar & 21 & 1,00 & $(0,63-1,50)$ \\
\hline Celulite orbitária & 9 & 0,43 & $(1,21-0,78)$ \\
\hline Hematoma periorbitário & 2 & 0,10 & $(0,02-0,31)$ \\
\hline Retina e vítreo & 29 & 1,38 & $(0,94-1,95)$ \\
\hline Descolamento de retina / Toxoplasmose & 23 & 1,09 & $(0,71-1,61)$ \\
\hline Hemorragia vítrea & 6 & 0,29 & $(0,16-0,59)$ \\
\hline Catarata & 21 & 1,00 & $(0,63-1,50)$ \\
\hline Glaucoma & 19 & 0,90 & $(0,56-1,38)$ \\
\hline Cefaléia & 13 & 0,62 & $(0,34-1,03)$ \\
\hline Exame normal & 28 & 1,33 & $(0,90-1,89)$ \\
\hline A esclarecer & 11 & 0,52 & $(0,28-0,91)$ \\
\hline
\end{tabular}

(*) um paciente pode ter mais de uma doença

Fonte: Prontuários médicos CEROF/UFG, 2009-2010

O registro de patologias sistêmicas nos indivíduos atendidos na urgência não foi expressivo. $\mathrm{O}$ diabetes mellitus foi registrado em 14 casos $(0,7 \%)$, a hipertensão arterial em 11 casos $(0,5 \%)$, problemas neurológicos em 4 casos $(0,2 \%)$ e outros em 5 casos $(0,2 \%)$, incluindo 1 caso de prematuridade.

\section{Discussão}

Entre os 2.105 prontuários de pacientes estudados, houve predominância do sexo masculino com resultados semelhantes encontrados por trabalhos em outros locais do Brasil
(1,2,5-9) e podem indicar uma maior exposição dos homens a fatores de risco, como traumas oculares no trabalho, esporte e trânsito. O mesmo ocorreu com a frequência de atendimento maior na faixa etária entre 15 e 39 anos, na urgência oftalmológica em relação a outras faixas etárias, confirmando serem os adultos jovens mais vulneráveis a traumas oculares. O inverso ocorreu com o grupo de pessoas com 60 anos ou mais que apresentou menor procura no atendimento de urgência.

Foram encontrados $1,3 \%$ da amostra de casos com exame oftalmológico normal. Provavelmente porque alguns casos não passam pela triagem médica do Hospital das Clínicas e outros 
são encaminhados pela triagem para avaliação do especialista e após exame não foram considerados casos de urgência.

Os grupos de doenças da córnea, conjuntiva e esclera $(71,63 \%)$ e das pálpebras e sistema lacrimal (17,96\%), tiveram maiores prevalências e foram seguidas pelo trauma ocular $(6,32 \%)$ e queimaduras químicas e térmicas $(4,13 \%)$.

No primeiro grupo houve predomínio de corpo estranho extraocular $(25,51 \%)$, conjuntivite infecciosa $(23,61 \%)$ e ceratites, englobando úlceras de córnea, $(11,26 \%)$ e o pterígio inclusive a pingueculite $(5,27 \%)$. Na pálpebra, predominaram tumores palpebrais $(7,22 \%)$, olho seco $(4,70 \%)$, blefarite $(3,75 \%)$ e triquíase $(0,95 \%)$ que na maioria das situações não são consideradas como urgência oftalmológica. Presume-se que as razões sejam as mesmas ao serem encontrados casos de exame normal nas consultas de urgência.

No sul do Brasil, Adamet al. ${ }^{(5)}$ referem predominância de conjuntivite aguda $(67,3 \%)$, corpo estranho de córnea $(13,2 \%)$, ceratites superficiais $(29 \%)$, pterígio $(13,2 \%)$ e pinguécula $(9,7 \%)$. As medidas foram feitas para a córnea e conjuntiva. Sugano et al. ${ }^{(7)}$ em hospital universitário registrou causas maiores de atendimento na urgência: doenças externas $(43,67 \%)$ com predomínio da conjuntivite $(54,61 \%)$ e trauma $(30,15 \%)$, com predomínio de corpo estranho extraocular (52,06\%). Campos Jr. ${ }^{(9)}$ encontrou em estudo populacional em clínica privada credenciada pelo SUS, maiores prevalências de conjuntivite aguda $(24,1 \%)$, corpo estranho extraocular $(17,9 \%)$ e trauma ocular mecânico ou químico (12,3\%), hipertensão ocular/glaucoma agudo $(5,7 \%)$, dor ocular inespecífica $(3,6 \%)$, uveíte $(3,2 \%)$,queimaduras oculares $(1,9 \%)$ e pterígio $(1,9 \%)$. Kara Jr et al. $(2001)^{(3)}$ em hospital de urgências oculares em São Paulo, relataram como causas mais comuns de atendimento na urgência a infecção $(34,0 \%)$, trauma ocular $(20 \%)$, tumores $(11,0 \%)$, descolamento de retina $(8,0 \%)$ e uveítes $(7,0 \%)$.

$\mathrm{Na}$ região nordeste do Brasil, Pierre Filho et al ${ }^{1}$ encontraram como maiores causas de atendimento trauma $(40,9 \%)$, infecções oculares $(29,0 \%)$ como conjuntivites, hordéolo e calázio e endoftalmites, pterígio $(4,0 \%) \mathrm{Na}$ córnea foram mais prevalentes: corpo estranho de córnea (53\%), ceratites / úlceras de córnea $(7,6 \%)$.

Vieira $^{(10)}$, em Brasília, encontrou como diagnósticos mais frequentes na urgência: traumas $(30 \%)$, conjuntivites $(24 \%)$, corpo estranho e outros traumas $(10 \%)$, ceratites $(6 \%)$, pterígio (5\%) e astenopia/exame normal (5\%).

Nos Estados Unidos, Mc Gwin et al. ${ }^{(11)}$ encontraram as ceratites superficiais, corpos estranhos como maiores prevalências de lesões oculares. Edwards ${ }^{(12)}$ encontrou na emergência uma predominância de adultos jovens (entre 20 a 39 anos), principalmente do sexo masculino, com maiores prevalências de traumas como abrasões e corpo estranho de córnea e conjuntiva e doenças inflamatórias como conjuntivite e blefarite.

Vários trabalhos citados apontam o trauma ocular como mais prevalente na urgência. No CEROF o trauma ocular ocupou o terceiro lugar de prevalência na urgência provavelmente casos de corpo estranho extraocular, hemorragia vítrea e ceratites e úlceras de córnea não foram citados como traumas nos prontuários, reduzindo a frequência relativa de casos.

Observou-se que uma parcela de pacientes procedida do interior do estado de Goiás e de outros estados, provavelmente pela escassez de locais prestadores deste tipo de atendimento à população economicamente carente, com conseqüente aumento da procura pelo CEROF. Muitos pacientes encaminhados são transportados pelas Prefeituras de origem, acarretando demora no atendimento que pode resultar em perda visual, além de aumento de custos assistenciais.
A frequência de atendimento na urgência do CEROF/ UFG(20\%) é alta em relação ao total de consultas comparada à observada em outros estudos brasileiros. Este fato pode-se dar devido à escassez de serviços credenciados pelo SUS que prestam este tipo de assistência, na Capital e região metropolitana, havendo a concentração de casos de urgência no serviço, aumentando proporcionalmente sua representação frente ao total de atendimentos de consultas (eletivas e urgência).

Muitos pacientes atendidos no serviço de urgência do CEROF-UFG apresentaram doenças comuns, de simples resolução, que poderiam ter sido diagnosticadas e tratadas em níveis primário e secundário de atendimento o que pode ser reflexo de falhas na rede de atendimento. O treinamento dos médicos destas Unidades através de cursos de Educação continuada aumentaria a resolução nestes níveis, evitando desgastes para o paciente e ganho de tempo além de reduzir a demanda excessiva na rede especializada, melhorando o atendimento dos casos que realmente necessitam do oftalmologista.

\section{REFERÊNCIAS}

1. Pierre Filho PTP, GomesPRP, Pierre ETL, Pinheiro NetoFB. Profile of ocular emergencies in a tertiary hospital from Northeast of Brazil. Rev Bras Oftalmol. 2010;69(1):12-7.

2. Cecchetti DFA, CecchettiSAP, NardyACT, Carvalho SC, Rodrigues MLV,Rocha EM. Perfil clínico e epidemiológico das urgências oculares em pronto-socorro de referência. Arq Bras Oftalmol. 2008;71(5):635-8

3. KaraJunior N, Zanatto MC, Villaça VTN, NagamatiLT, KaraJoséN. Aspectos médicos e sociais no atendimento oftalmológico de urgência. Arq Bras Oftalmol. 2001;64(1):39-43.

4. GoiatoMC, MancusoDN, FernandesAUR,Dekon SFC. Estudo sobre as causas mais freqüentes de perdas oculares. Arq Odontol.2004;40(3):271-6.

5. Adam Netto AA, Rolim APQ, Müller TPS. Prevalência de doenças palpebrais no serviço emergencial de oftalmologia do Hospital Universitário da Universidade Federal de Santa Catarina.ACM ArqCatarinMed. 2006;35(4):64-9.

6. Adam NettoA, SiewertMC, Müller TPS, Silvano RE, Thiesen EB, Queiroz AA. Prevalência de doenças corneanas no serviço emergencial de oftalmologia do Hospital Universitário da Universidade Federal de Santa Catarina. ACM ArqCatarinMed. 2006;35(4):50-5.

7. Sugano DM, Ávila MP, Lima VL, Carvalho F, Rehder JRCL. Estudo do perfil de demanda e morbidade ocular em um serviço deemergência oftalmológica no período de 1999 a 2002. Rev BrasOftalmol.2004;63(4):231-5.

8. Araújo AA, Almeida DV, Araújo VM, GóesMR. Urgência oftalmológica: corpo estranho ocular ainda como principal causa. Arq BrasOftalmol.2002;65(2):223-7.

9. Campos JúniorJC. Perfil do atendimento oftalmológico de urgência. Rev Bras Oftalmol. 2004;63(2):89-91.

10. VieiraGM. Um mês em um pronto-socorro de oftalmologia em Brasília. ArqBras Oftalmol. 2007;70(5):797-802

11. McGwin G Jr,XieA, Owsley C. Rate of eye injury in the United States. ArchOphthalmol.2005;123(7):970-6.Erratum in Arch Ophthalmol. 2005;123(9):1285.

12. Edwards RS. Ophthalmic emergencies in a district general hospital casualty department. Br JOphthalmol.1987;71(12):938-42.

\section{Autor correspondente:}

Maria Nice Araujo Moraes Rocha

Rua 12, no 345, apto. 1402

CEP 74 140-040 - Setor Oeste - Goiânia - (GO), Brasil

E-mail: clinicarocha@bol.com.br 